\title{
Spontaneously hypertensive rats do not predict symptoms of attention-deficit hyperactivity disorder
}

\author{
Filip S. van den Bergh*, Emilie Bloemarts, Johnny S.W. Chan, \\ Lucianne Groenink, Berend Olivier, Ronald S. Oosting \\ Utrecht Institute for Pharmaceutical Sciences, and Rudolf Magnus Institute of Neuroscience Department of Psychopharmacology, \\ Utrecht University, Sorbonnelaan 16, 3584 CA Utrecht, The Netherlands
}

Received 27 May 2005; received in revised form 15 February 2006; accepted 18 February 2006

Available online 6 March 2006

\begin{abstract}
The validity of the Spontaneously Hypertensive rat (SHR) as a model for Attention Deficit Hyperactivity Disorder (ADHD) is explored by comparing the SHR with Wistar-Kyoto (WKY) and Wistar rats in a number of different tests. In the open field, SHR are hyperactive compared to both Wistar and WKY, but only at specific ages. At those ages, methylphenidate $(1 \mathrm{mg} / \mathrm{kg})$ did not attenuate hyperactivity. Subsequently, a dose response study of methylphenidate $(0.1-10 \mathrm{mg} / \mathrm{kg}$ ) was conducted in the Differential Reinforcement of Low-rate responding (DRL)-72s and fivechoice serial reaction time tests (5-CSRTT). Compared to WKY but not Wistar rats, SHR performed worse on the DRL-72s. Performance was not improved by methylphenidate $(0.1-1.0 \mathrm{mg} / \mathrm{kg})$. In the 5-CSRTT, attentional performance was similar for all rat strains, but Wistar rats made more impulsive responses than both the SHR and the WKY. Methylphenidate only attenuated impulsivity in Wistar rats. Because SHR do not consistently display symptoms of ADHD across the different tests, and methylphenidate effects were observed in both WKY and Wistar rats, but not in SHR, we conclude that SHR is not a representative animal model for ADHD.
\end{abstract}

(C) 2006 Elsevier Inc. All rights reserved

Keywords: Spontaneously hypertensive rat; Animal model; Methylphenidate; Differential reinforcement of low-rate responding; Five-choice serial reaction time task; Open field; Extinction; Attention-deficit hyperactivity disorder

\section{Introduction}

Attention deficit hyperactivity disorder (ADHD) is a complex CNS disorder characterized by hyperactivity, inattention and impulsivity. The disorder is diagnosed in $4-12 \%$ of 6 - to $12-$ year-old children, and symptoms may persist into adulthood (Resnick, 2005; Wender, 2002; Wolraich et al., 1998). ADHD is usually treated with methylphenidate or comparable mild psychomotor stimulants. Although $70-90 \%$ of patients respond to such treatment, there are several drawbacks to using stimulant medication. First, stimulants can induce insomnia, anorexia, headache, and stomach-problems. Second, there is a potential for abuse, and not much is known about long-term effects. And third, methylphenidate has a short half-life, further limiting its use (Bolanos et al., 2003; Goldman et al., 1998). To discover

\footnotetext{
* Corresponding author. Tel.: +31 30253 7383; fax: +31 302537387 .

E-mail address: F.S.vandenBergh@pharm.uu.nl (F.S. van den Bergh).
}

new drug targets involved in processes underlying ADHD, animal models are indispensable. Because the diagnosis of ADHD itself is not without controversy, modeling ADHD in animals is difficult. Nevertheless, different animal models for ADHD have been proposed (Davids et al., 2003), of which the spontaneously hypertensive rat (SHR) seems the best validated model. This rat strain seems to display all three symptoms of ADHD: hyperactivity, an attention deficit, and impulsivity (Sagvolden, 2000). In addition, several biochemical differences between the SHR and the normotensive controls are reminiscent of ADHD. SHR differ from controls in the dopamine system, including an altered response to psychostimulants (Oades, 2002; Russell et al., 1998, 2000b; Volkow et al., 2001), an increased noradrenergic activity (Russell et al., 2000a), and a decreased serotonergic functioning (Kulikov et al., 1997; Nakamura et al., 2001). The use of the SHR as a model for ADHD, however, is not without problems. Although the SHR and WKY were derived from the same colony of outbred Wistar rats, they were 
derived at different times (WKY after SHR) and therefore the WKY are not exactly the normotensive genetic analog of the SHR (Okamoto, 1969), and an increasing number of studies address the validity of the WKY rat as the control animal for the SHR. The WKY is known for its inactivity, leading to an exaggeration of the ADHD-like symptoms in the SHR. In fact, the WKY is very susceptible to learned helplessness, and has even been proposed as a model for depression (Wieland et al., 1986; Will et al., 2003). When both WKY and SHR are compared to other often-used rat strains, it seems that the SHR is not so much hyperactive, but the WKY is particularly inactive instead (Bull et al., 2000; Pare, 1989; Sagvolden et al., 1993).

The usefulness of the SHR as a model for ADHD, however, is not defined by the resemblance of its behavior to the symptoms of ADHD alone. Predictions about characteristics of ADHD resulting from experiments using the SHR should correspond to findings in the patient population, so the animal model may be used to test putative new medications (Geyer and Markou, 1995). Although necessary, predictive validity is not sufficient for a good animal model. Specifically, methylphenidate, the treatment of choice for patients suffering from ADHD, should have a similar alleviating effect in the SHR and in the patient population (Aron et al., 2003). Although there have been more studies on the effects of methylphenidate in the SHR, only few have tested the animal model in more widely validated tasks for attention and impulsivity, such as the five-choice serial reaction time task (5CSRTT), and the differential reinforcement of low-rate responding (DRL) task (see for example Evenden and Meyerson, 1999; Sagvolden et al., 1992b, 1993).

The goal of the present experiment was to assess the validity of the SHR as a model for ADHD in several well-validated tests for activity, attention and impulse control. The frequently used Wistar rat served as a control in addition to the WKY, to counteract the previously described problems associated with the WKY strain as a control strain. Furthermore, to assess the predictive validity of the SHR as a model for ADHD, the effects of methylphenidate on activity, attention and impulsivity have been measured.

\section{Methods}

\subsection{Experimental design}

In Experiment 1, Wistar rats were used to establish a methylphenidate dose range using the five-choice serial reaction time task. In Experiment 2, both male and female rats of all three strains were repeatedly tested for locomotor hyperactivity in an open field test at age 30 days, 44 days, 58 days, 72 days, 86 days, and 100 days. In Experiment 3, another group (at age 30-44 days because the previous experiment indicated that the differences between the SHR and the other strains were largest at that age) consisting of all three strains was tested in the open field, now under administration of methylphenidate. In Experiment 4, half of the animals used for Experiment 3 started the acquisition-reversal-extinction battery, used to measure general operant ability of the animals because most tests for attention and impulsivity are operant tasks. Changes in such basic operant behavior may underlie changes found in the performance on other, more complex tasks, such as those used in Experiments 5 and 6. After the acquisition-reversalextinction battery, rats progressed to Experiment 5, and were trained in the DRL-72s task, a test that has been in use for over 40 years (Richards et al., 1993; Woolverton and Alling, 1999). In this task, animals are rewarded for pressing a lever-but only if the previous response was more than $72 \mathrm{~s}$ previously. Experiment 6, the 5CSRTT, was conducted on the other half of the animals used in experiment three (Carli et al., 1983; Robbins, 2002). In this task, animals are rewarded food pellets for responding to brief flashes of light in one of five holes. The accuracy of responding serves as a measure for attention. Criterion performance for experiments five and six was reached at approximately the same time, and the effects of $0.1,1$ and $10 \mathrm{mg} / \mathrm{kg}$ methylphenidate (p.o., $60 \mathrm{~min}$ before testing) on DRL and the 5CSRTT performance were tested.

\subsection{Subjects}

The study was composed of six experiments conducted in three separate groups of rats. In the first experiment, 20 male Wistar rats (HsdCpb:WU) obtained from Harlan (The Netherlands) were used for an initial methylphenidate dose-response study. In the remaining experiments three different strains were used: Wistar rats (HsdCpb:WU), Wistar-Kyoto rats (WKY/ NHsd), and Spontaneously Hypertensive Rats (SHR/NHsd). In the second experiment, 8 male and 8 female rats of the three strains were tested in the open field at different ages. The rats were bred at the Faculty's animal facility from parents obtained from Harlan (United States). For the third experiment, 16 male rats of each of the three strains were used. The Wistar rats were bred at Harlan's facilities in the Netherlands, while Harlan imported both the 16 SHR as well as the 16 Wistar Kyoto rats from their United States facility. For the fourth, fifth and sixth experiment, the same animals were used as in experiment 3.

All animals were housed in groups of four of identical strain in a light (lights on from 7:00 AM to 7:00 PM), temperature (21 $\left.\pm 2{ }^{\circ} \mathrm{C}\right)$ and humidity $(55 \pm 5 \%)$ controlled facility. All tests were conducted during the light phase. To motivate the rats (except those used in experiment 2) to respond during the operant tasks, they were on a diet of $15 \mathrm{~g}$ of standard laboratory chow per day and were rewarded food pellets for proper responses in the operant tasks. Animal weight corresponded to $85 \pm 5 \%$ of freefood weight. Water was freely available. The animals were weighed and checked for health problems weekly by a veterinary technician. The ethical committee on animal experiments of the Faculties of Pharmaceutical Sciences, Chemistry and Biology of Utrecht University, The Netherlands, approved the experiments.

\subsection{Apparatus}

The open field used in the second experiment was different from the open field used in the third experiment. In the second experiment, open field tests were conducted in four square gray PVC containers measuring $75 \mathrm{~cm}$ by $75 \mathrm{~cm}$ and $40 \mathrm{~cm}$ tall. In the third experiment, cylinders of $45 \mathrm{~cm}$ in diameter and $30 \mathrm{~cm}$ tall 
were used. The movements of the animals in the open field were tracked over $60 \mathrm{~min}$ via a video-system in real-time using Noldus Ethovision 3 (Noldus et al., 2001).

Eight Skinner boxes (MED Associates) were used, all equipped with a food magazine, delivering $45 \mathrm{mg}$ Noyes precision pellets Formula P (Research Diets, New Brunswick, USA). The operant chambers were controlled by MED-PC IV software. Four of those eight boxes were used for the acquisition-reversalextinction tests and the DRL procedure, and were equipped with retractable levers to the left and right of the food magazine. The remaining four boxes were used for the 5CSRTT, and were equipped with a five-hole nose poke wall opposite to the food magazine. In each of these holes, a light stimulus could be presented, and nose poke responses could be registered. In addition, motion detectors were mounted on the ceiling in these boxes.

\subsection{Open field}

In the second experiment, animals were placed in the open field untreated. In the third experiment, $1 \mathrm{~h}$ before each open field test, animals (around age 30 days) were either injected with saline or $1 \mathrm{mg} / \mathrm{kg}$ methylphenidate (p.o.). The animals remained in the open field for $60 \mathrm{~min}$. After the session, all subjects were returned to their home cage.

\subsection{Acquisition-reversal-extinction battery}

At the start of each acquisition session, both levers were extended into the operant cage. Subjects received a food reward every time they pressed the left lever only. If subjects received 25 pellets in a 30-min session, they reached criterion, and received no more acquisition sessions. Reversal sessions were similar to acquisition sessions, but in these sessions the animals had to press the lever not reinforced in the previous session. All animals received two reversal sessions. During the extinction sessions, both levers were again extended, but pressing the levers yielded no food reward. All animals received five extinction sessions.

\section{6. $D R L-72 s$}

Only animals that were included in the acquisition-reversalextinction battery were trained on the DRL schedule. Since these animals had just received extinction training, they had to be retrained to press the right lever for food reward. The right lever was the only extended lever during the DRL training. After initial retraining, animals received 1-h DRL training sessions daily. In these sessions, only responses that were more than $6 \mathrm{~s}$ after the previous response were rewarded. The minimum inter-response time was increased over the course of the training until the target inter-response time of $72 \mathrm{~s} \mathrm{had}$ been reached $(6 \mathrm{~s}, 12 \mathrm{~s}, 24 \mathrm{~s}, 36 \mathrm{~s}, 48 \mathrm{~s}, 60 \mathrm{~s}, 72 \mathrm{~s})$.

When subjects reached the target inter-response time, they were injected with methylphenidate one hour prior to the test. The dosages $(0.1 \mathrm{mg} / \mathrm{kg}, 1.0 \mathrm{mg} / \mathrm{kg}$ and $10 \mathrm{mg} / \mathrm{kg})$ were administered according to a within-subjects Latin-square design. Drug tests were conducted in two consecutive weeks on Tuesday and Thursday.

\subsection{Five-choice serial reaction time task}

In phase 1, animals were habituated to the operant chamber by putting them in the operant chamber for $15 \mathrm{~min}$, while 15 pellets were freely available in the food magazine. Phase 2 habituated animals to the feeder over the course of two 20-min sessions where food was dispensed every $20 \mathrm{~s}$. In phase 3 animals were trained to make nose pokes in one of the lit apertures of the 5-hole wall. During this phase, the same aperture was lit all the time. Animals reached criterion performance if they made more than 50 nose pokes during a 20 -min session. Then, the final training phase commenced. Sessions of this type lasted either an hour, or until 100 trials had been initiated. To start a trial, animals were required to make a nose poke in the food magazine. After the variable (5-10s) inter-trial interval, a randomly selected aperture was lit, and animals were rewarded for responses in that aperture either during the presentation of the stimulus, or during the limited hold period that followed presentation. The stimulus presentation times were decreased during training $(30 \mathrm{~s}, 10 \mathrm{~s}, 5 \mathrm{~s}, 2 \mathrm{~s}, 1 \mathrm{~s})$, while the limited hold period was fixed at $5 \mathrm{~s}$. Responses into any other aperture than the target or a failure to respond at all were punished by a timeout. During a timeout, the house light was extinguished for $5 \mathrm{~s}$, after which a magazine nose poke initiated the next trial. For the first experiment (the dose-response study) and the first part of the second experiment (the strain comparison), anticipatory responses were punished by timeout. To increase the chances of finding an effect of methylphenidate, anticipatory responses were no longer punished during the second part of the training for the second experiment (Hahn et al., 2002).

If the subjects' performance at the target stimulus duration (1 s) was adequate (\%correct $>60 \%, \%$ omissions $<15 \%$ ), animals were injected with methylphenidate one hour prior to the test. The criteria were obtained by inspecting the performance daily until no further improvement was made. The dosages $(0.1 \mathrm{mg} / \mathrm{kg}, 1.0 \mathrm{mg} / \mathrm{kg}$ and $10 \mathrm{mg} / \mathrm{kg})$ were administered according to a within-subjects Latin-square design. Drug tests were conducted twice per week on two consecutive weeks on Tuesday and Thursday.

\subsection{Data reduction and analysis}

All data were analyzed using the SPSS General Linear Model procedure. Variables with a not-normal distribution as determined by visual inspection and by the Kolmogorov-Smirnov test were translated using a log-transformation. Significant differences $(p<0.05)$ were further explored using Bonferroni corrected post-hoc comparisons. For strain differences, all strains were compared. For drug effects, comparisons to vehicle conditions were made and $p$-values were multiplied by the number of non-vehicle administrations to correct for multiple comparisons.

In the open field, drug administration and open field session were entered into the general linear model as a between-subjects variable, while the time served as a within-subjects variable. In the DRL and the 5CSRTT, drug administration was analyzed as a within-subjects variable. 
The acquisition-reversal-extinction battery was analyzed in three parts. First, the acquisition times were compared. Then, the number of responses on the reinforced lever compared to the number of non-reinforced responses was compared for the three strains. Finally, the three strains were compared by the number of responses in the five extinction sessions.

Statistical analysis of responding in the DRL was not restricted to the number of obtained rewards. The responses during the session were collected in $6 \mathrm{~s}$ bins, allowing for the calculation of the burst ratio (the number of responses made in the $0-6 \mathrm{~s}$ after a previous response compared to the total number of responses), the peak area and the peak location. An elaborate description of this method is provided by (Richards et al., 1993).

Correct and incorrect responses in the 5CSRTT were calculated as a percentage of the total number of trials that ended in nose poke. Anticipatory responses and omissions however, were calculated as a percentage of all initiated trials. Other measures included in the analysis were activity as measured by the ceilingmounted motion detectors, number of magazine responses (sometimes taken as a measure for activity) and the latency to collect rewards (sometimes taken as a measure for motivation), make correct responses and to make incorrect responses.

\subsection{Drugs}

To closely simulate clinical administration, methylphenidate hydrochloride derived from RITALIN tablets (Novartis Pharma, Arnhem) were used. Tablets containing $10 \mathrm{mg}$ of methylphenidate were crushed and suspended in gelatin $(0.5 \%)$ /mannitol $(5 \%)$. Suspensions were freshly made every morning, and vortexed before every administration. Ground tablets have not been used before in such research, and because ground tablets (including additives) administered orally may have different pharmacokinetic properties due to a slower absorption rate, we conducted a dose-response study to establish an optimal dose range. First, $5 \mathrm{mg} / \mathrm{kg}, 10 \mathrm{mg} / \mathrm{kg}$ and $20 \mathrm{mg} / \mathrm{kg}$ were tested followed by a lower range: $0.5 \mathrm{mg} / \mathrm{kg}, 2.5 \mathrm{mg} / \mathrm{kg}$ and $5 \mathrm{mg} / \mathrm{kg}$ (p.o., at $60 \mathrm{~min}$ before testing). Based on those results, the dose range for the strain comparisons was determined to be $0.1 \mathrm{mg} / \mathrm{kg}$, $1.0 \mathrm{mg} / \mathrm{kg}$ and $10 \mathrm{mg} / \mathrm{kg}$. At the highest dose, dopamine levels in the nucleus accumbens have been shown to more than double after an hour (Gerasimov et al., 2000). Although this dose range is not identical to ranges used in other studies (Thai et al., 1999), the pilot study indicated the range to be optimal for oral administration of ground tablets, allowing for the measurement of both stimulating (high dose) and calming (low dose) effects of methylphenidate. Oral administrations were performed by a skilled technician in freely moving animals.

\section{Results}

\subsection{Experiment 1: Methylphenidate in the five-choice serial reaction time task}

The effects of oral administration of methylphenidate on premature responding in the 5CSRTT were dependent on dose (see Fig. 1). In the first experiment using high dosages $(5,10$,
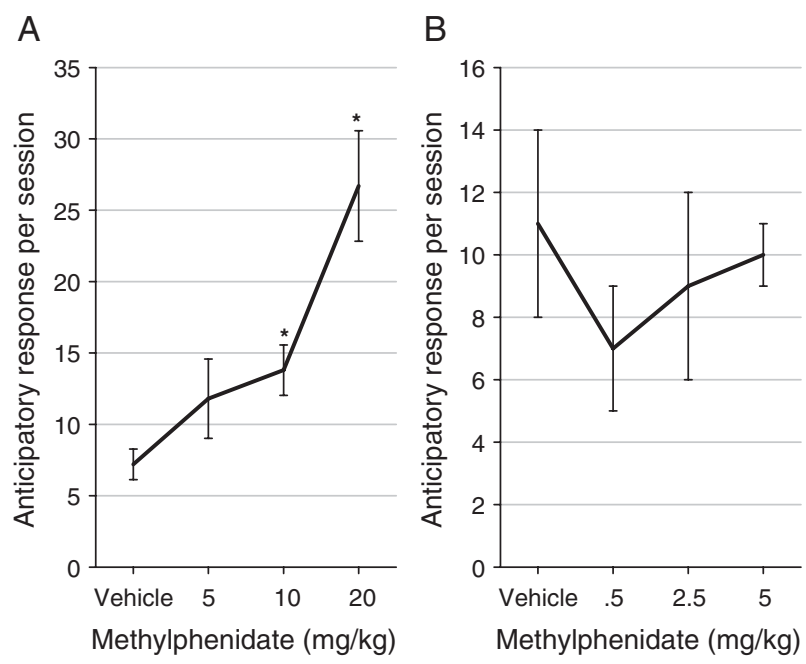

Fig. 1. Effects of methylphenidate in two dose ranges on anticipatory responses in the 5CSRTT. Values are cumulative mean responses \pm SEM. Significant effects of methylphenidate are indicated with $*(p<0.05)$.

$20 \mathrm{mg} / \mathrm{kg}$ ), methylphenidate significantly elevated anticipatory responding $(F[3,51]=13.0, p<0.005)$. This effect was significant at $10 \mathrm{mg} / \mathrm{kg}$ and $20 \mathrm{mg} / \mathrm{kg} \quad(p<0.05$ and $p<0.001$, respectively). In the second experiment using lower dosages, methylphenidate also significantly altered anticipatory responding $(F[3,8]=5.0, p<0.05)$. Although this decrease was most pronounced $(41 \%)$ at $0.5 \mathrm{mg} / \mathrm{kg}$, it did not reach statistical significance. For further experimentation, a dose range was chosen that incorporated both slightly lower and slightly higher dosages $(0.1 \mathrm{mg} / \mathrm{kg}, 1.0 \mathrm{mg} / \mathrm{kg})$, as well as a dose that resulted in an increase of anticipatory responses $(10 \mathrm{mg} / \mathrm{kg}$ ). In addition, it was decided to use an adapted version of the 5CSRTT would be used to maximize the effects of methylphenidate. If anticipatory responses go unpunished by timeout, the test becomes more sensitive to the effects of psychostimulants (Hahn et al., 2002).

\subsection{Experiment 2: Effects of gender and age on open field activity}

As can be seen in Fig. 2, the development of activity was different for the three strains (age $\times$ strain interaction: $F$ $[3.5,195]=3.5, p<0.005)$. As a result of the complex interactions, not one strain can be said to be more active than the others across the entire 100 days (main effect of strain: $F[2,39]=2.9$, ns). At age 30 days, however, male SHR are more active than WKY (separate ANOVA: $F[2,41]=4.6, p<0.005$, SHR vs. WKY: $p<0.05$ ). Because of the SHRs hyperactivity at that time, 30-day-old animals were also used in Experiment 3. Hyperactivity of the SHR was not found in female rats.

\subsection{Experiment 3: Effects of methylphenidate on open field activity}

The activity of the three strains on the three consecutive open field tests with and without administration of $1 \mathrm{mg} / \mathrm{kg}$ methylphenidate is depicted in Fig. 3. Across all three sessions, SHR were significantly more active in the open field than 

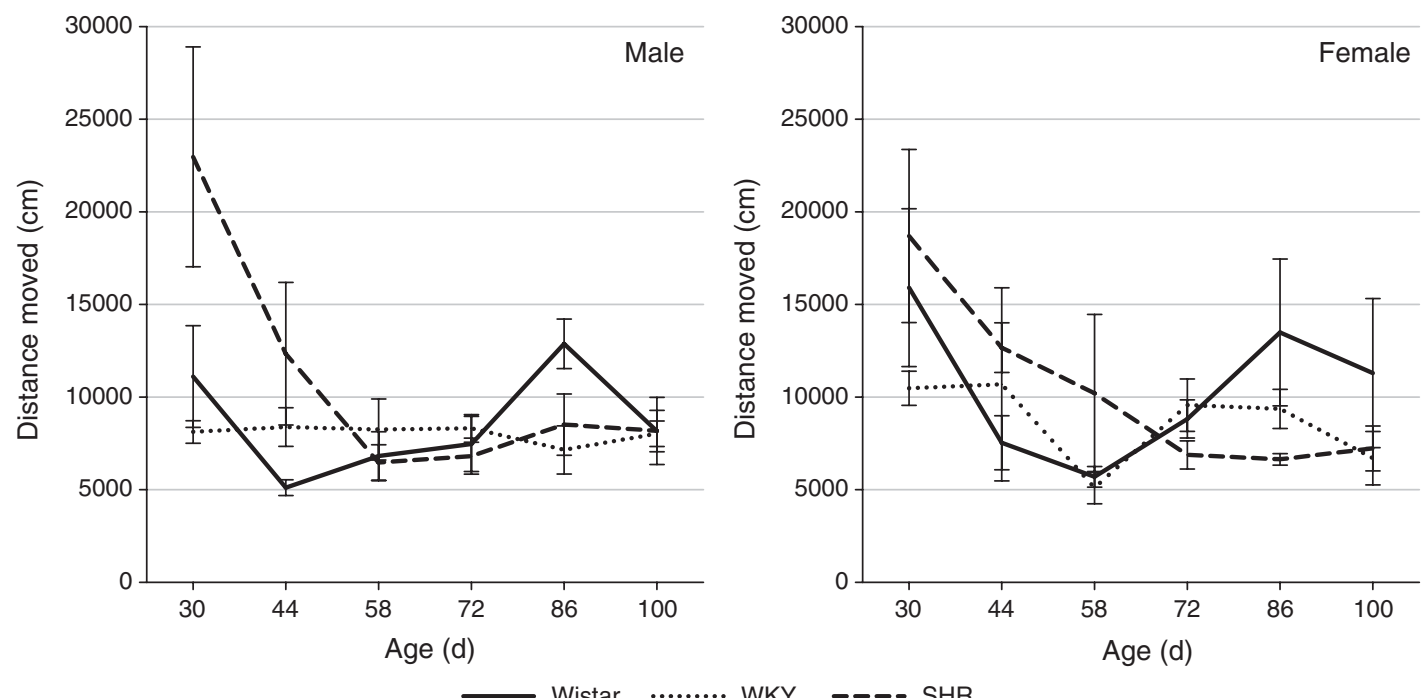

Fig. 2. Development of open field activity over six different ages. Distance moved in the open field by male rats is shown in the left graph while activity of female rats is shown in the right graph. Values are total distance moved over $60 \mathrm{~m} \pm$ SEM. Male SHR are significantly more active at 30 days, while Wistar rats are more active at 86 days.
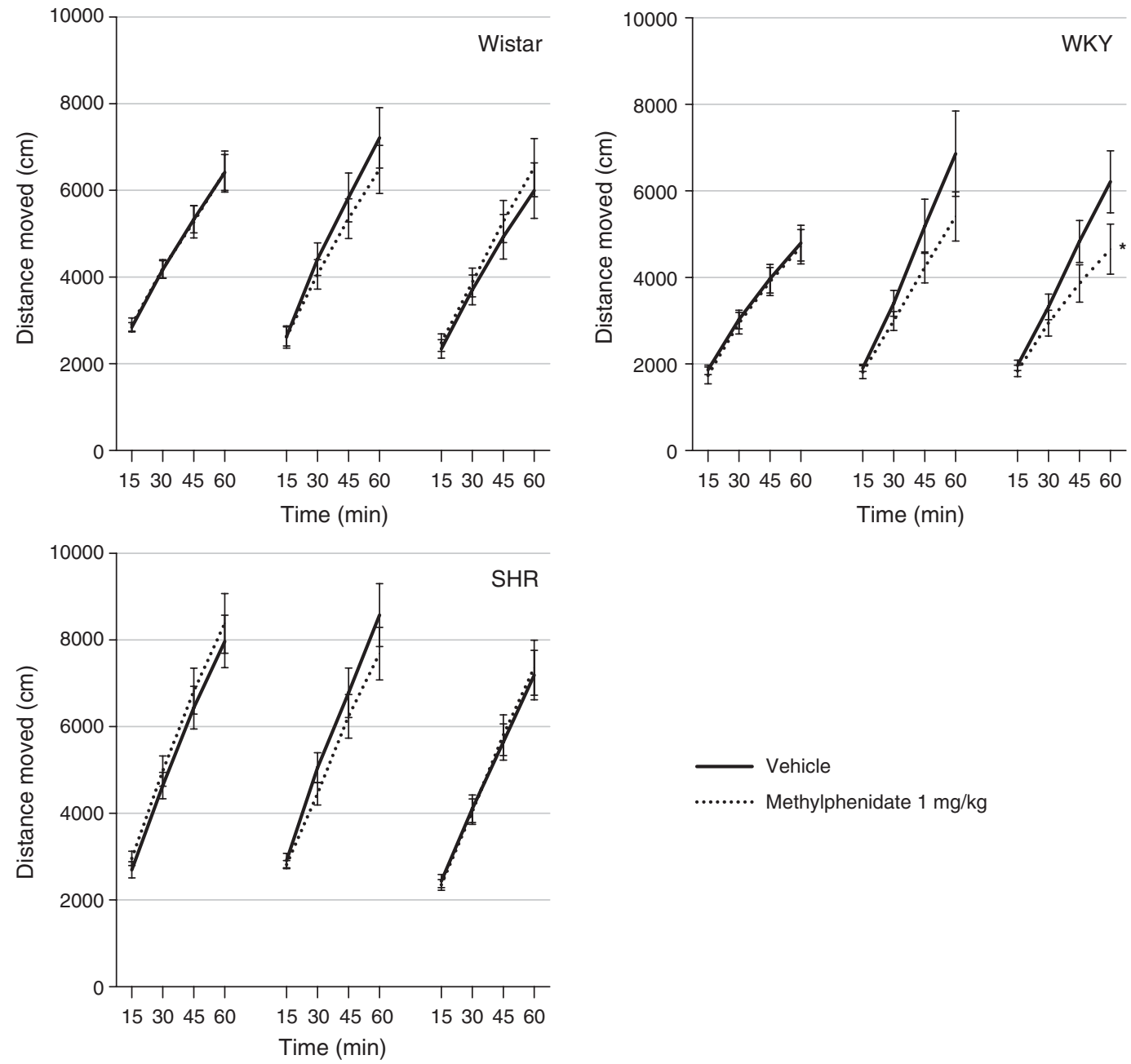

Fig. 3. Effects of strain and methylphenidate on cumulative locomotor activity in three consecutive sessions (once per week, starting at age 30 days) of the open field test. Treatments across the three sessions were always the same. Values are cumulative distance \pm SEM. Significant effects of methylphenidate are indicated with * $(p<0.05)$ 
Wistar Kyoto rats (main effect: $F[2,22]=9.5, p<0.005$, SHR vs. WKY: $p<0.005$ ). The difference between WKY and Wistar rats was almost significant ( $p=0.055$ ), whereas activity of SHR and Wistar rats did not differ.

Although the treatment $\times$ strain interaction was not significant $(F[2,22]=2.6, p=0.1)$, treatment effects were calculated separately for each strain. Both the SHR and the normal Wistar rat showed no effect of the $1 \mathrm{mg} / \mathrm{kg}$ dose of methylphenidate administration $(F<1$ in both cases). The activity of the WKY rat, however, was suppressed by methylphenidate (treatment main effect $F[1,8]=6.6, p<0.05$; treatment $\times$ open field session interaction $F[2,7]=4.7, p=0.05$ ). Further exploring this suppression shows that it was particularly apparent in the third open field test (where $F[1,11]=6.2, p<0.05$ ).

\subsection{Experiment 4: Acquisition-reversal-extinction battery}

The time to acquire lever-pressing did not differ for the three strains (mean \pm SEM: Wistar: $27 \pm 6.8 \mathrm{~min}, \mathrm{WKY}: 55 \pm 15.2 \mathrm{~min}$, SHR: $38 \pm 6.4 \mathrm{~min} ; F[2,20]=1.8$, NS). The number of responses on the non-reinforced lever during the two reversal sessions was also not different across groups $(F[2,20]=1.2$, NS for the first reversal, $F[2,20]<1$ for the second reversal session). Although the WKY rats lagged behind during the session, the session completion time was not significantly longer $(F[2,20]=2.7$, $p=0.092$ ). During the five extinction sessions (Fig. 4), the SHR responded significantly more than both other strains, and the Wistar rats made significantly more responses than the WKY rats (main effect: $F[2,19]=26.9, p<0.001$, SHR vs. WIS: $p<0.001$, SHR vs. WKY: $p<0.001$, WIS vs. WKY: $p<0.05$ ).

\subsection{Experiment 5: DRL-72s}

The number of rewards obtained and the burst ratio in the DRL is depicted in Fig. 5. The other parameters of
A

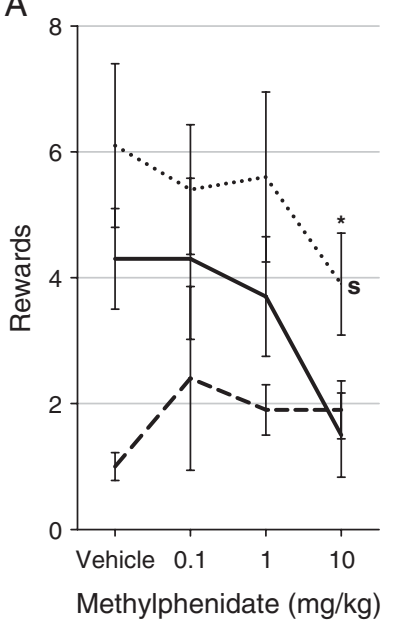

B

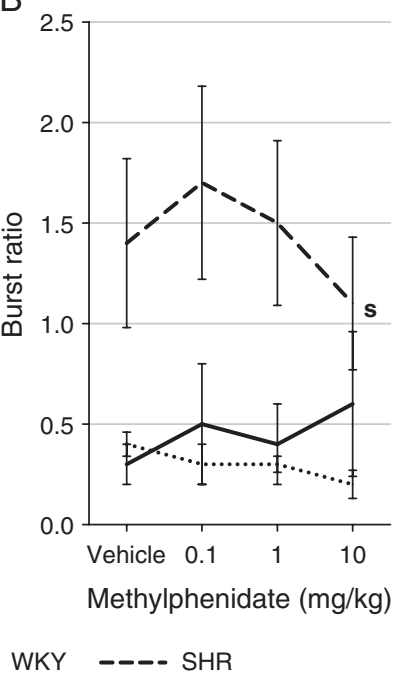

Fig. 5. Rewards (A) and burst ratios (B) in the DRL-72s. Values are group means \pm SEM. Significant differences are indicated with 's' (for a significant strain difference $p<0.05$ ) and * (for a significant drug effect $p<0.05$ ).

the DRL (peak latency and peak area) are summarized in Table 1. One Wistar rat was removed from the analysis because it performed too well ( 26 rewards obtained while the Wistar group mean was 4.3 rewards, Cook's distance was $0.95)$.

SHR received significantly fewer rewards than WKY rats (main effect: $F[2,18]=5.3, p<0.05$, SHR vs. WKY: $p<0.05$ ). The mean number of rewards obtained by the Wistar rats was lower than the mean for the WKY rats, but higher than the mean for the SHR, resulting in no significant differences. Administration of methylphenidate led to a significant overall decrease in rewards obtained $(F[3,16]=3.6, p=0.038)$, post hoc tests confirmed that $10 \mathrm{mg} / \mathrm{kg}$ methylphenidate resulted in a decrease

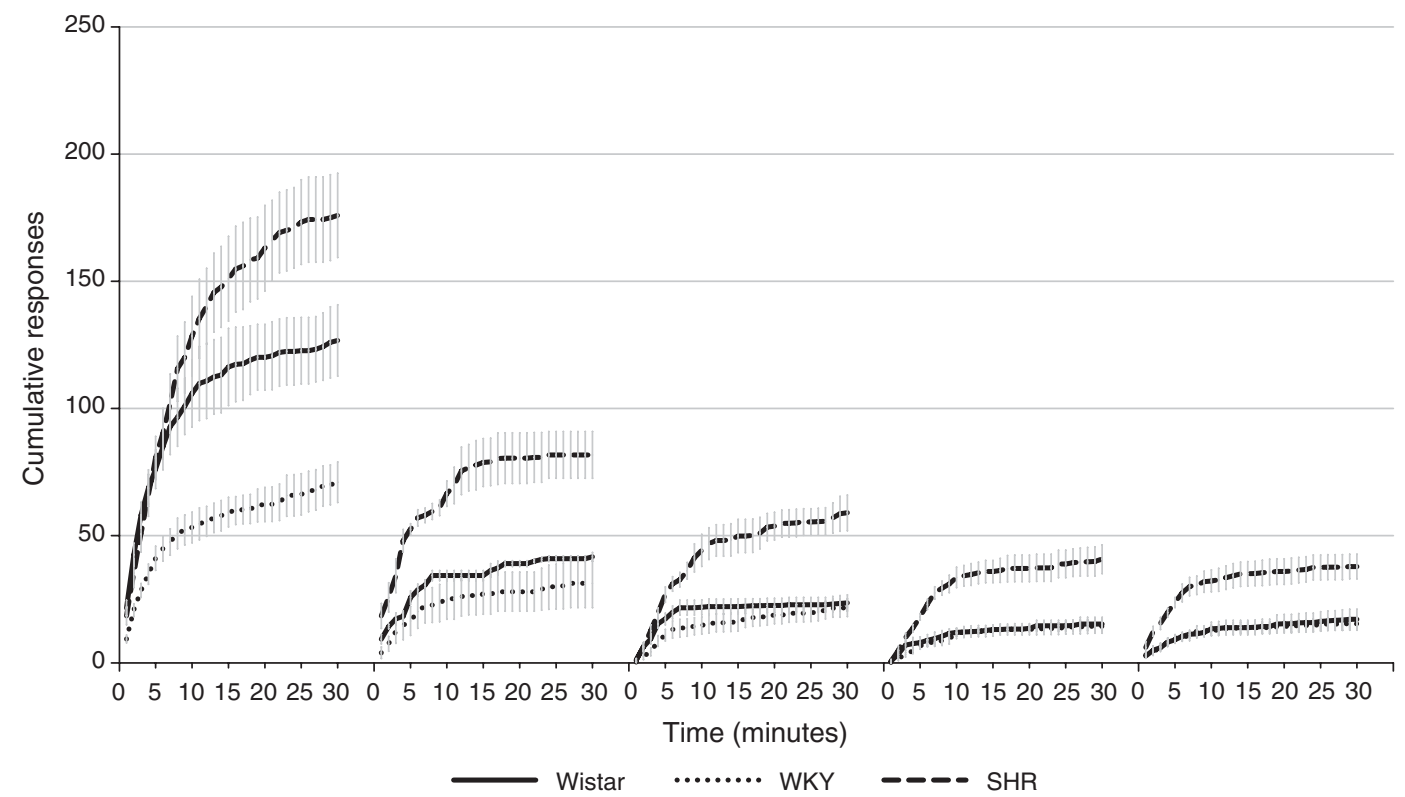

Fig. 4. Number of responses during five consecutive extinction sessions. None of the levers yielded rewards. Values are cumulative mean responses \pm SEM. 
Table 1

DRL peak analysis parameters

\begin{tabular}{lcccc}
\hline & Vehicle & $0.1 \mathrm{mg} / \mathrm{kg}$ & $1.0 \mathrm{mg} / \mathrm{kg}$ & $10 \mathrm{mg} / \mathrm{kg}$ \\
\hline Wistar rats & & & & \\
Peak latency*d & $31.2 \pm 8.2$ & $33.9 \pm 5.8$ & $32.9 \pm 6.0$ & $30.3 \pm 5.6$ \\
Peak area & $0.3 \pm 0.1$ & $0.3 \pm 0.04$ & $0.3 \pm 0.03$ & $0.4 \pm 0.1$ \\
& & & & \\
Wistar-Kyoto rats & & & & \\
Peak latency*d & $35.8 \pm 3.3$ & $36.1 \pm 5.1$ & $38.2 \pm 2.7$ & $27.6 \pm 2.0$ \\
Peak area & $0.4 \pm 0.03$ & $0.4 \pm 0.04$ & $0.4 \pm 0.04$ & $0.4 \pm 0.03$ \\
& & & & \\
Spontaneously hypertensive rats & & & \\
Peak latency*d & $23.8 \pm 2.1$ & $29.4 \pm 3.5$ & $26.4 \pm 2.5$ & $21.9 \pm 2.5$ \\
Peak area & $0.3 \pm 0.03$ & $0.4 \pm 0.04$ & $0.4 \pm 0.03$ & $0.3 \pm 0.04$ \\
\hline
\end{tabular}

The table values indicate scores \pm standard error of the mean.

*s Significant strain effect $p<0.05$.

${ }^{*}$ Significant drug effect $p<0.05$.

in rewards obtained compared to the vehicle condition $(p<0.05)$.

Burst responding was largely dependent on strain $(F[2,18]=$ $7.0, p<0.01)$. The dose $\times$ strain interaction was also significant $(F[6,34]=3.0, p<0.05)$. SHR show up to $600 \%$ higher burst ratios than other strains (SHR vs. WKY: $p<0.01$, SHR vs. Wistar: $p<0.05$ ). Burst ratios of WKY and Wistar rats did not differ significantly. To further explore the dose $\times$ strain interaction, the dose effects were analyzed separately for each strain. The effects of methylphenidate on burst responding were strongest in the SHR although the results did not reach significance $(F[3,4]=5.4, p=0.069)$. There were neither strain nor dose differences on the peak area $(F[2,18]<1, F[3,16]<1$, respectively).

The main effect of strain on peak latency did not reach statistical significance $(F[2,18]=3.1,0.069)$, but the peak latency was sensitive to methylphenidate $(F[3,16]=4.4$, $p<0.05)$. A post-hoc analysis revealed no differences between dosages.
Table 2

Miscellaneous parameters for the five-choice serial reaction time task

Vehicle $\quad 0.1 \mathrm{mg} / \mathrm{kg} \quad 1.0 \mathrm{mg} / \mathrm{kg} \quad 10 \mathrm{mg} / \mathrm{kg}$

\begin{tabular}{lrrrr}
\hline Wistar rats & & & & \\
Time to complete session (min)*s & $24.3 \pm 0.6$ & $24.8 \pm 0.4$ & $25.3 \pm 1.2$ & $24.7 \pm 0.6$ \\
\%Incorrect during stimulus & $21.5 \pm 4.5$ & $20.9 \pm 6.3$ & $18.5 \pm 5.4$ & $24.4 \pm 7.6$ \\
\%Incorrect during limited hold & $9.1 \pm 1.6$ & $9.0 \pm 1.2$ & $9.4 \pm 1.1$ & $7.5 \pm 2.0$ \\
\%Omissions & $5.1 \pm 1.1$ & $6.4 \pm 2.1$ & $7.0 \pm 1.8$ & $7.7 \pm 2.6$ \\
\%Perseveratives & $1.4 \pm 1.0$ & $1.0 \pm 0.5$ & $0.7 \pm 0.3$ & $0.9 \pm 0.3$ \\
Magazine responses & $9.4 \pm 0.7$ & $9.7 \pm 0.9$ & $9.6 \pm 1.0$ & $9.3 \pm 1.0$
\end{tabular}

Wistar-Kyoto rats

$\begin{array}{lllll}\text { Time to complete session }(\min ) * \mathrm{~s} & 29.8 \pm 1.0 & 36.2 \pm 4.0 & 30.3 \pm 2.0 & 37.2 \pm 3.9\end{array}$

$\begin{array}{lllll}\text { \%Incorrect during stimulus } & 17.6 \pm 4.2 & 18.0 \pm 5.5 & 16.8 \pm 3.6 & 17.5 \pm 5.1\end{array}$

$\begin{array}{lllll}\text { \%Incorrect during limited hold } & 9.6 \pm 1.4 & 7.7 \pm 1.6 & 9.7 \pm 1.0 & 9.3 \pm 1.3\end{array}$

$\%$ Omissions

\%Perseveratives

$11.6 \pm 2.5 \quad 12.7 \pm 3.1 \quad 9.8 \pm 2.2 \quad 11.1 \pm 2.5$

$\begin{array}{llll}0.9 \pm 0.3 & 0.9 \pm 0.2 & 1.0 \pm 0.4 & 0.7 \pm 0.1\end{array}$

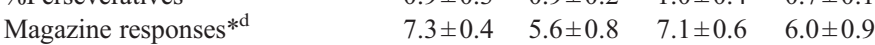

Spontaneously hypertensive rats

$\begin{array}{lllll}\text { Time to complete session }(\mathrm{min}) * \mathrm{~s} & 32.5 \pm 1.8 & 34.1 \pm 1.7 & 33.7 \pm 1.2 & 37.3 \pm 3.9\end{array}$

$\begin{array}{lllll}\text { \%Incorrect during stimulus } & 25.7 \pm 4.0 & 27.8 \pm 5.2 & 27.0 \pm 4.0 & 26.1 \pm 5.2\end{array}$

$\begin{array}{lrrrr}\text { \%Incorrect during limited hold } & 12.5 \pm 2.9 & 10.6 \pm 1.7 & 12.2 \pm 1.6 & 18.2 \pm 6.5\end{array}$

$\%$ Omissions

\%Perseveratives

$\begin{array}{llll}9.6 \pm 3.3 & 8.6 \pm 3.4 & 9.1 \pm 3.6 & 18.5 \pm 9.7\end{array}$

$\begin{array}{lllll}\text { Magazine responses*d }^{* d} & 11.5 \pm 0.7 & 10.6 \pm 1.0 & 9.6 \pm 0.8 & 8.6 \pm 1.3\end{array}$

The table values indicate scores \pm standard error of the mean.

${ }^{*}$ Significant strain effect $p<0.05$.

${ }^{* \mathrm{~d}}$ Significant drug effect $p<0.05$.

\subsection{Experiment 6: Five-choice serial reaction time task}

Fig. 6 shows the accuracy of responding, the number of anticipatory responses and the activity in the 5CSRTT. Other five-choice measures are listed in Table 2.

WKY rats took significantly longer to reach criterion than both the SHR and the Wistar rats (mean \pm SEM: Wistar: $9 \pm 0.4$ sessions, WKY: $16 \pm 1.9$ sessions, SHR: $11 \pm 1.0$ sessions; main effect: $F[3,21]=7.9, p<0.005$, SHR vs. WKY: $p<0.005$, SHR
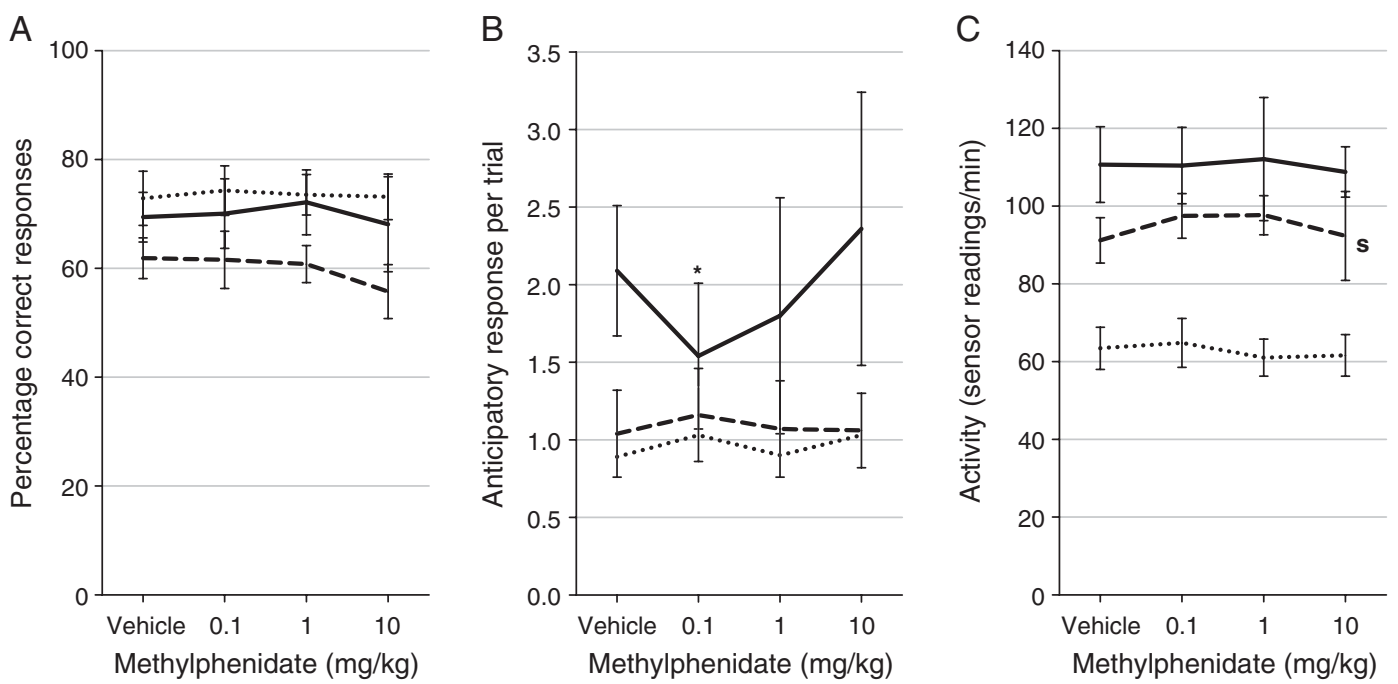

- Wistar ........ WKY ---- SHR

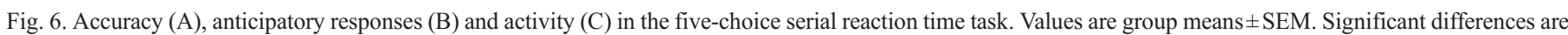
indicated with ' $\mathrm{s}$ ' (for a significant strain difference $p<0.05$ ) and * (for a significant drug effect $p<0.05$ ). 
vs. Wistar: $p<0.05)$. SHR were about as fast to acquire the task as Wistar rats. Correct responses in the 5CSRTT did not depend on strain $(F[3,18]<1)$. Because the dose $\times$ strain interaction bordered significance for the anticipatory responses $(F[3,18]=$ 2.2, $p=0.061)$, the strain differences were calculated only for the vehicle group, and the drug differences were analyzed for each group separately. The main effect of strain was significant $(F[2,20]=4.2, p=0.031)$, due to the difference between the Wistar rats (the group that scored the most anticipatory responses) and the WKY rats $(p<0.05)$. The higher number of anticipatory responses of the Wistar rats did not differ statistically from the level of the SHR $(p=0.088)$. The effects of methylphenidate were present only in the Wistar group, where the main effect of dose was significant $(F[3,4]=18, p<0.01)$. Post hoc comparisons show that $0.1 \mathrm{mg} / \mathrm{kg}$ significantly lowered impulsivity compared to vehicle $(p<0.05)$. Activity in the operant cage as measured by ceiling-mounted motion detectors was dependent on strain $(F[2,20]=13.2, p<0.001)$. Both the SHR and the Wistar rats were significantly more active than the WKY rats ( $p<0.001$ for both comparisons). The SHR were as active as the Wistar rats $(p=0.11)$.

Session duration was dependent on strain $(F[2,20]=18.8$, $p<0.001)$. Wistar rats completed the session faster than both SHR and WKY rats (Wistar vs. SHR: $p<0.001$, Wistar vs. WKY: $p<0.001)$. The time to complete the session was not different for SHR and WKY rats. Incorrect responses during stimulus presentation and during the limited hold were not affected by strain $(F[3,18]<1$ for both). The slightly higher level of omissions of the WKY rats was also not significant $(F$ $[3,18]<1)$. Because of a tendency towards a dose $\times$ strain interaction $(F[6,38]=2.0, p=0.089)$, the data were analyzed separately. For the SHR and the WKY rats, a decrease of magazine responses was measured with increasing dosages of methylphenidate. This decrease did not reach statistical significance $(F[3,5]=4, p=0.085$ and $F[3,5]=3.7, p=0.098$, respectively). For the Wistar rats, there was no such pattern $(F$ $[3,4]<1)$. The latency to collect obtained rewards, the latency to make a correct response and the latency to make an incorrect response were no different for each of the three groups (main effects: $F[3,18]=1.0, \mathrm{NS}, F[3,18]<1, F[3,18]<1$ respectively).

\section{Discussion}

The aim of the present research was to validate the SHR as a model for ADHD. SHR were more active in the open field than WKY rats, but only at specific ages. Furthermore, this activity was not normalized by methylphenidate, only WKY were less active with methylphenidate administration. Acquisition and reversal of lever pressing was not different, but SHR did show slower extinction. SHR made more burst responses and received fewer rewards in the DRL, but methylphenidate did not normalize their performance. SHR did not show an attention deficit in the 5CSRTT, and were less impulsive than Wistar rats. Only impulsivity of Wistar rats was alleviated by methylphenidate.

The diagnostic and statistical manual (DSM-IV) describes the first symptom, hyperactivity, as an inability to remain seated or keep quiet, and as often on the go. In animals, activity is most frequently measured in the open field. Open field behavior of the three strains has often been studied, although the data are not very consistent. SHR are sometimes more active than control strains (Hard et al., 1985), while sometimes control strains are more active (Ferguson et al., 2003; Sagvolden et al., 1993). This hyperactivity is not always present at the first session (e.g. Knardahl and Sagvolden, 1979), and sometimes psychomotor stimulants suppress this activity (Myers et al., 1982; Wultz et al., 1990). In the present study, only young (30 days old) SHR traveled much more than WKY. At the same age, Wistar rats traveled about as much as the SHR, but the pattern of activity over an hour in the open field is different. Although Wistar rats are as active as SHR in the first $15 \mathrm{~min}$ of the test, in the remaining $45 \mathrm{~min}$, Wistar rats are about as active as WKY rats. The different origins of the animals cannot account for this difference, as the results of animals bred in our facility (Experiment 2) and imported animals (Experiment 3) are very similar. Why do juvenile SHR keep exploring the open field? Previous studies have shown that SHR do not habituate to novel environments as fast as WKY do (Hendley et al., 1985). Are they, like hippocampally damaged animals, unable to remember where they have been (Good and Honey, 1997)? Performance of SHR in the Morris water maze suggest they do not have a worse spatial memory, and may even exhibit faster spatial orientation than WKY and Sprague-Dawley rats (Diana, 2002; Ferguson and Cada, 2004). Perhaps the SHR rats are less anxious than the other two strains. Indeed, 74-day-old SHR display less anxietyrelated behavior in the elevated plus maze (Ferguson and Gray, 2005). Anxious WKY rats may habituate to the open field with repeated exposure, and may therefore also explain why WKY rats display increased locomotor activity in the third session of Experiment 3. Whatever the cause of locomotor hyperactivity in the SHR, this increase is not attenuated by administration of methylphenidate. In contrast, activity of the WKY is attenuated by methylphenidate. Although it would be interesting to study why this hyperactivity disappears over time, the insensitivity to methylphenidate suggests that this process is unrelated to ADHD.

Comparing our results to literature, we conclude that SHR show badly replicable performance in the open field (Ferguson et al., 2003; Hard et al., 1985; Knardahl and Sagvolden, 1979; Myers et al., 1982; Sagvolden et al., 1993; Wultz et al., 1990). Based on replicability and pharmacology, we conclude the SHR in the open field is not a useful model for ADHD.

As a measure of the second symptom of ADHD, an attention deficit, the 5CSRTT was used, an operant task in which animals are trained to detect brief flashes of light. The accuracy of responding reflects attention, while the ability to withhold responses until a stimulus has been presented measures impulsivity. In line with previous research, SHR perform no different from Wistar controls (De Bruin et al., 2003). WKY rats show a similar level of accuracy, but required more sessions to reach criterion performance. This slower acquisition may be related to the inactivity of the WKY rats, which was also reflected in the activity levels as measured by the motion detectors. Because the SHR were not less accurate, and because 
methylphenidate did not result in increased attention, SHR in the 5CSRTT do not seem to be a good model for ADHD. In addition, the present results add to the notion that the WKY rats' inactivity makes it an unsuitable control.

The final symptom of ADHD is impulsivity. Impulsive responses are rapid responses made without much forethought or deliberation (Evenden, 1999). Patients fail to delay their responses to the appropriate moment: they give answers before questions are completed or are unable to wait their turn in games. To test for impulsivity in rats, the DRL and the 5CSRTT were used.

In the DRL, animals are rewarded food pellets for pressing a lever $72 \mathrm{~s}$ after their previous lever response. The SHR and the WKY have been tested in this paradigm before, using the Sprague-Dawley strain as an additional control (Bull et al., 2000). There have not been many reports of the effects of methylphenidate in the DRL, but other psychomotor stimulants generally worsen performance (Balcells-Olivero et al., 1997, 1998; Sabol et al., 1995). In the present study, SHR received less rewards than the WKY controls, but not compared to the Wistar rats. In accordance with the lower amount of rewards, the peak location for the response distribution curve of the SHR was shifted towards the left. In addition, they made more burst responses than both control groups. These results matched reports of performance of SHR in the DRL (Bull et al., 2000). In addition to that replication, we extended those results by measuring the effects of methylphenidate in all three strains. The first effect of methylphenidate was a decrease in rewards consistent with earlier reports (Seiden et al., 1979). In contrast to both control strains, the performance of SHR was unaffected by methylphenidate, even though it allowed more room for improvement. The second effect of methylphenidate was on burst responses. Unfortunately, no post-hoc comparisons were significant, probably due to the small number of animals. How should we interpret the elevated burst responding of the SHR, and the effects of methylphenidate on these fast responses? Perhaps the same neural mechanism that led to burst responding in the DRL led to persistent responding during extinction sessions in the acquisition-reversal-extinction battery. During the extinction sessions, SHR pressed the non-reinforced levers as much as twice more than the Wistar controls. The link between burst responses in the DRL and extinction is supported by significant correlations (Pearson's correlation coefficient $r$ ) between the burst ratio and the number of responses during the five extinction sessions ranging from 0.52 (in the fourth extinction session; $p<0.01$ ) to 0.81 (in the fifth extinction session; $p<0.01$ ). Because of the association between extinction and burst responding, the slower extinction is more likely due to motor hyperactivity rather than deficient memory. Slower extinction can experimentally be induced by serotonergic depletion (Beninger and Phillips, 1979), a procedure that can also lead to an increase in burst responses (Jolly et al., 1999). Persistence during the extinction sessions and elevated burst responses in the SHR are probably related to serotonergic hypofunctioning in the striatum (Nakamura et al., 2001).

The 5CSRTT provided a second measure of impulsivity: the anticipatory responses made before the stimulus was presented. Contrary to the DRL findings, the SHR were not the most impulsive group. Previous research also reported the SHR is no more impulsive than control groups (De Bruin et al., 2003). In addition, in the present experiment, the more impulsive Wistar rats responded to methylphenidate by a decrease in impulsivity, while the other strains did not. The impulsivity-attenuating effect of methylphenidate was in accordance with literature (Bizarro et al., 2004), and more pronounced than the data from the initial methylphenidate dose-response study. The increase of effectiveness of methylphenidate compared to the initial dose-response study may be due to the anticipatory responses not being punished by timeout (Hahn et al., 2002). In the present study, SHR did not display lowered impulsivity at the same dose or any other dose. The cause of this failure may be due to altered methylphenidate metabolism in SHR (in particular a possible faster clearance), but this will remain speculation without further research.

It is unclear why the 5CSRTT measure for impulsivity is different from the DRL data and the extinction data, although changes made to the 5CSRTT may be responsible: in the present experiment, anticipatory responses were not punished by timeout. Although this may have increased our chances of finding an effect of methylphenidate, this may also have decreased our chances of finding a strain effect and concordance with the burst ratio. Anticipatory responses in the adapted version of the 5CSRTT task may be a measure of a different type of impulsivity than the burst responses in the DRL (Evenden, 1999). Performance in the DRL may be more related to motor inhibitory control (Pattij et al., 2003), while impulsive responses in the 5CSRTT may be a measurement of a more general concept of impulsivity.

In the DRL, the SHR are more impulsive, but this task was not sensitive to methylphenidate. The 5CSRTT, on the other hand, was sensitive to methylphenidate, but did not indicate the SHR as more impulsive. Deciding what is the right test for measuring impulsivity is difficult, but neither aids in distinguishing the usefulness of the SHR as a model for ADHD. Either the 5CSRTT is chosen and there is no impulsivity effect, or the DRL is chosen and the predictive validity cannot be established.

This research adds to a growing body of work indicating that SHR are not a reliable, nor a readily reproducible, model for ADHD (Bull et al., 2000; Ferguson and Cada, 2003). Several findings indicate that performance of the SHR is very different from performance of WKY controls, but there are major problems with this comparison. When the SHR is compared to Sprague-Dawley rats or to Wistar rats such as in the present research, the WKY appears to be a very inactive and nonimpulsive animal compared to other widely used rat strains, and may be unsuited as a control (Bull et al., 2000; Diana, 2002; Pare, 1989; Sagvolden et al., 1993). In addition, the lack of efficacy of methylphenidate at the tested dosages for any of the domains of ADHD further limits the usefulness of the SHR. Because methylphenidate was effective in several other tests and strains in the present research, this lack of efficacy cannot be attributed to the used dosages.

There is some evidence that SHR can be divided into two subpopulations, one impulsive and one normal (Adriani et al., 
2003). The division into subgroups was not tested extensively, however, as the resulting subgroups were very small. Therefore, before accepting that hypothesis much more research is necessary to make sure that this distinction is not an artifact. Furthermore, the effects of psychostimulants in delayed reward paradigms (a shift towards the large but delayed reward) found in the SHR (Adriani and Laviola, 2004) is not specific to that strain (Cardinal et al., 2000). This raises more questions about the usefulness of the SHR as a model.

Although we conclude that the SHR is not a good model for ADHD, this may not be the result of the specific strains used, but rather a more fundamental problem of using two inbred strains as a model for psychiatric illness. A comparison between two strains will often result in a difference on several behavioral measures. Sometimes these differences are valued as to represent a human condition. The number of modeled symptoms is often used as a criterion of model quality (for example, see Sagvolden, 2000), but each of these traits may be caused by a different mechanism. For example, in the case of the SHR, the genetic cause of hypertension is unrelated to inattentiveness, impulsivity or hyperactivity (Hendley, 2000; Sagvolden et al., 1992a). The co-occurrence of hypertension with impulsivity is therefore not predictive of ADHD. Likewise, all other differences between SHR and WKY may be attributed to separate genetic origins as well, as SHR and WKY are known to be very different genetically (Festing and Bender, 1984; St Lezin et al., 1992).

\section{References}

Adriani W, Laviola G. Windows of vulnerability to psychopathology and therapeutic strategy in the adolescent rodent model. Behav Pharmacol 2004; $15: 341-52$.

Adriani W, Spijker S, Deroche-Gamonet V, Laviola G, Le Moal M, Smit AB, et al. Evidence for enhanced neurobehavioral vulnerability to nicotine during periadolescence in rats. J Neurosci 2003;23:4712-6.

Aron AR, Dowson JH, Sahakian BJ, Robbins TW. Methylphenidate improves response inhibition in adults with attention-deficit/hyperactivity disorder. Biol Psychiatry 2003;54:1465-8.

Balcells-Olivero M, Richards JB, Seiden LS. Sensitization to amphetamine on the differential-reinforcement-of-low-rate 72-s schedule. Psychopharmacology (Berl) 1997;133:207-13.

Balcells-Olivero M, Cousins MS, Seiden LS. Holtzman and Harlan SpragueDawley rats: differences in DRL 72-sec performance and 8-hydroxy-dipropylamino tetralin-induced hypothermia. J Pharmacol Exp Ther 1998;286:742-52.

Beninger RJ, Phillips AG. Possible involvement of serotonin in extinction. Pharmacol Biochem Behav 1979;10:37-41.

Bizarro L, Patel S, Murtagh C, Stolerman IP. Differential effects of psychomotor stimulants on attentional performance in rats: nicotine, amphetamine, caffeine and methylphenidate. Behav Pharmacol 2004;15:195-206.

Bolanos CA, Barrot M, Berton O, Wallace-Black D, Nestler EJ. Methylphenidate treatment during pre- and periadolescence alters behavioral responses to emotional stimuli at adulthood. Biol Psychiatry 2003;54:1317-29.

Bull E, Reavill C, Hagan JJ, Overend P, Jones DN. Evaluation of the spontaneously hypertensive rat as a model of attention deficit hyperactivity disorder: acquisition and performance of the DRL-60s test. Behav Brain Res 2000;109:27-35.

Cardinal RN, Robbins TW, Everitt BJ. The effects of d-amphetamine, chlordiazepoxide, alpha-flupenthixol and behavioural manipulations on choice of signalled and unsignalled delayed reinforcement in rats. Psychopharmacology (Berl) 2000;152:362-75.
Carli M, Robbins TW, Evenden JL, Everitt BJ. Effects of lesions to ascending noradrenergic neurones on performance of a 5-choice serial reaction task in rats; implications for theories of dorsal noradrenergic bundle function based on selective attention and arousal. Behav Brain Res 1983;9:361-80.

Davids E, Zhang K, Tarazi FI, Baldessarini RJ. Animal models of attentiondeficit hyperactivity disorder. Brain Res Brain Res Rev 2003;42:1-21.

De Bruin NM, Kiliaan AJ, De Wilde MC, Broersen LM. Combined uridine and choline administration improves cognitive deficits in spontaneously hypertensive rats. Neurobiol Learn Mem 2003;80:63-79.

Diana G. Does hypertension alone lead to cognitive decline in spontaneously hypertensive rats? Behav Brain Res 2002;134:113-21.

Evenden JL. Varieties of impulsivity. Psychopharmacology (Berl) 1999; 146:348-61.

Evenden J, Meyerson B. The behavior of spontaneously hypertensive and Wistar Kyoto rats under a paced fixed consecutive number schedule of reinforcement. Pharmacol Biochem Behav 1999;63:71-82.

Ferguson SA, Cada AM. A longitudinal study of short- and long-term activity levels in male and female spontaneously hypertensive, Wistar-Kyoto, and Sprague-Dawley rats. Behav Neurosci 2003;117:271-82.

Ferguson SA, Cada AM. Spatial learning/memory and social and nonsocial behaviors in the Spontaneously Hypertensive, Wistar-Kyoto and SpragueDawley rat strains. Pharmacol Biochem Behav 2004;77:583-94.

Ferguson SA, Gray EP. Aging effects on elevated plus maze behavior in spontaneously hypertensive, Wistar-Kyoto and Sprague-Dawley male and female rats. Physiol Behav 2005;85:621-8.

Ferguson SA, Gray EP, Cada AM. Early behavioral development in the spontaneously hypertensive rat: a comparison with the Wistar-Kyoto and Sprague-Dawley strains. Behav Neurosci 2003;117:263-70.

Festing MF, Bender K. Genetic relationships between inbred strains of rats. An analysis based on genetic markers at 28 biochemical loci. Genet Res 1984;44:271-81.

Gerasimov MR, Franceschi M, Volkow ND, Gifford A, Gatley SJ, Marsteller D, et al. Comparison between intraperitoneal and oral methylphenidate administration: A microdialysis and locomotor activity study. J Pharmacol Exp Ther 2000;295:51-7.

Geyer MA, Markou A. Animal models of psychiatric disorders. In: Bloom FE, Kupfer DJ, Bloom FE, Kupfer DJs, editors. Psychopharmacology: the fourth generation of progress. New York: Raven Press, Ltd.; 1995. p. 787-98.

Goldman LS, Genel M, Bezman RJ, Slanetz PJ. Diagnosis and treatment of attention-deficit/hyperactivity disorder in children and adolescents. Council on Scientific Affairs American Medical Association. JAMA 1998; 279:1100-7.

Good M, Honey RC. Dissociable effects of selective lesions to hippocampal subsystems on exploratory behavior, contextual learning, and spatial learning. Behav Neurosci 1997;111:487-93.

Hahn B, Shoaib M, Stolerman IP. Nicotine-induced enhancement of attention in the five-choice serial reaction time task: the influence of task demands. Psychopharmacology (Berl) 2002;162:129-37.

Hard E, Carlsson SG, Jern S, Larsson K, Lindh AS, Svensson L. Behavioral reactivity in spontaneously hypertensive rats. Physiol Behav 1985; 35:487-92.

Hendley ED. WKHA rats with genetic hyperactivity and hyperreactivity to stress: a review. Neurosci Biobehav Rev 2000;24:41-4.

Hendley ED, Wessel DJ, Atwater DG, Gellis J, Whitehorn D, Low WC. Age, sex and strain differences in activity and habituation in SHR and WKY rats. Physiol Behav 1985;34:379-83.

Jolly DC, Richards JB, Seiden LS. Serotonergic mediation of DRL 72s behavior: receptor subtype involvement in a behavioral screen for antidepressant drugs. Biol Psychiatry 1999;45:1151-62.

Knardahl S, Sagvolden T. Open-field behavior of spontaneously hypertensive rats. Behav Neural Biol 1979;27:187-200.

Kulikov A, Aguerre S, Berton O, Ramos A, Mormede P, Chaouloff F. Central serotonergic systems in the spontaneously hypertensive and Lewis rat strains that differ in the elevated plus-maze test of anxiety. J Pharmacol Exp Ther 1997;281:775-84.

Myers MM, Musty RE, Hendley ED. Attenuation of hyperactivity in the spontaneously hypertensive rat by amphetamine. Behav Neural Biol 1982; $34: 42-54$. 
Nakamura K, Shirane M, Koshikawa N. Site-specific activation of dopamine and serotonin transmission by aniracetam in the mesocorticolimbic pathway of rats. Brain Res 2001;897:82-92.

Noldus LP, Spink AJ, Tegelenbosch RA. EthoVision: a versatile video tracking system for automation of behavioral experiments. Behav Res Methods Instrum Comput 2001;33:398-414.

Oades RD. Dopamine may be 'hyper' with respect to noradrenaline metabolism, but 'hypo' with respect to serotonin metabolism in children with attentiondeficit hyperactivity disorder. Behav Brain Res 2002;130:97-102.

Okamoto K. Spontaneous hypertension in rats. Int Rev Exp Pathol 1969; 7:227-70.

Pare WP. Stress ulcer and open-field behavior of spontaneously hypertensive, normotensive, and Wistar rats. Pavlov J Biol Sci 1989;24:54-7.

Pattij T, Broersen LM, van der Linde J, Groenink L, van der Gugten J, Maes RA, et al. Operant learning and differential-reinforcement-of-low-rate 36-s responding in 5-HT1A and 5-HT1B receptor knockout mice. Behav Brain Res 2003;141:137-45.

Resnick RJ. Attention deficit hyperactivity disorder in teens and adults: They don't all outgrow it. J Clin Psychol 2005;61(5):529-33 [May].

Richards JB, Sabol KE, Seiden LS. DRL interresponse-time distributions: quantification by peak deviation analysis. J Exp Anal Behav 1993; $60: 361-85$.

Robbins TW. The 5-choice serial reaction time task: behavioural pharmacology and functional neurochemistry. Psychopharmacology (Berl) 2002; 163:362-80.

Russell V, de Villiers A, Sagvolden T, Lamm M, Taljaard J. Differences between electrically-, ritalin- and D-amphetamine-stimulated release of [3H]dopamine from brain slices suggest impaired vesicular storage of dopamine in an animal model of Attention-Deficit Hyperactivity Disorder. Behav Brain Res 1998;94:163-71.

Russell V, Allie S, Wiggins T. Increased noradrenergic activity in prefrontal cortex slices of an animal model for attention-deficit hyperactivity disorderthe spontaneously hypertensive rat. Behav Brain Res 2000a;117:69-74.

Russell VA, de Villiers AS, Sagvolden T, Lamm MC, Taljaard JJ. Methylphenidate affects striatal dopamine differently in an animal model for attention-deficit/hyperactivity disorder-the spontaneously hypertensive rat. Brain Res Bull 2000b;53:187-92.

Sabol KE, Richards JB, Layton K, Seiden LS. Amphetamine analogs have differential effects on DRL 36-s schedule performance. Psychopharmacology (Berl) 1995;121:57-65.

Sagvolden T. Behavioral validation of the spontaneously hypertensive rat (SHR) as an animal model of attention-deficit/hyperactivity disorder (AD/HD). Neurosci Biobehav Rev 2000;24:31-9.
Sagvolden T, Hendley ED, Knardahl S. Behavior of hypertensive and hyperactive rat strains: hyperactivity is not unitarily determined. Physiol Behav 1992a;52:49-57.

Sagvolden T, Metzger MA, Schiorbeck HK, Rugland AL, Spinnangr I, Sagvolden G. The spontaneously hypertensive rat (SHR) as an animal model of childhood hyperactivity (ADHD): changed reactivity to reinforcers and to psychomotor stimulants. Behav Neural Biol 1992b;58:103-12.

Sagvolden T, Pettersen MB, Larsen MC. Spontaneously hypertensive rats (SHR) as a putative animal model of childhood hyperkinesis: SHR behavior compared to four other rat strains. Physiol Behav 1993;54:1047-55.

Seiden LS, Andresen J, MacPhail RC. Methylphenidate and d-amphetamine: effects and interactions with alphamethyltyrosine and tetrabenazine on DRL performance in rats. Pharmacol Biochem Behav 1979;10:577-84.

St Lezin E, Simonet L, Pravenec M, Kurtz TW. Hypertensive strains and normotensive 'control' strains. How closely are they related? Hypertension 1992;19:419-24.

Thai DL, Yurasits LN, Rudolph GR, Perel JM. Comparative pharmacokinetics and tissue distribution of the d-enantiomers of para-substituted methylphenidate analogs. Drug Metab Dispos 1999;27:645-50.

Volkow ND, Wang G, Fowler JS, Logan J, Gerasimov M, Maynard L, et al. Therapeutic doses of oral methylphenidate significantly increase extracellular dopamine in the human brain. J Neurosci 2001;21:RC121.

Wender EH. Attention-deficit/hyperactivity disorder: is it common? Is it overtreated? Arch Pediatr Adolesc Med 2002;156:209-10.

Wieland S, Boren JL, Consroe PF, Martin A. Stock differences in the susceptibility of rats to learned helplessness training. Life Sci 1986; 39:937-44.

Will CC, Aird F, Redei EE. Selectively bred Wistar-Kyoto rats: an animal model of depression and hyper-responsiveness to antidepressants. Mol Psychiatry 2003;8:925-32.

Wolraich ML, Hannah JN, Baumgaertel A, Feurer ID. Examination of DSM-IV criteria for attention deficit/hyperactivity disorder in a county-wide sample. $\mathrm{J}$ Dev Behav Pediatr 1998;19:162-8.

Woolverton WL, Alling K. Choice under concurrent VI schedules: comparison of behavior maintained by cocaine or food. Psychopharmacology (Berl) 1999;141:47-56.

Wultz B, Sagvolden T, Moser EI, Moser MB. The spontaneously hypertensive rat as an animal model of attention-deficit hyperactivity disorder: effects of methylphenidate on exploratory behavior. Behav Neural Biol 1990; 53:88-102. 